\title{
Cognitive Fatigability Interventions in Neurological Conditions: A Systematic Review
}

\author{
Lisa A. S. Walker (D) - Alyssa P. Lindsay-Brown · Jason A. Berard
}

Received: July 30, 2019 / Published online: October 4, 2019

(c) The Author(s) 2019

\begin{abstract}
Introduction: Although fatigue is a well-studied concept in neurological disease, cognitive fatigability (CF) is less understood. While most studies measure fatigue using subjective self-report, fewer have measured CF objectively. Given the negative impact of CF on quality-oflife, there is a need for targeted interventions. The objective of this review was to determine which procedural, behavioural and pharmacological treatments for objectively measured CF are available to people living with neurological conditions.
\end{abstract}

Methods: In accordance with the PRISMA guidelines, systematic searches for randomized

Enhanced Digital Features To view enhanced digital features for this article go to https://doi.org/10.6084/ m9.figshare.9885368.

Electronic supplementary material The online version of this article (https://doi.org/10.1007/s40120019-00158-3) contains supplementary material, which is available to authorized users.

L. A. S. Walker $(\bowtie) \cdot$ A. P. Lindsay-Brown .

J. A. Berard

Ottawa Hospital Research Institute, Ottawa, Canada

e-mail: lwalker@toh.ca

L. A. S. Walker

University of Ottawa Brain and Mind Research

Institute, Ottawa, Canada

L. A. S. Walker

Carleton University, Ottawa, Canada control trials (RCTs), case-controlled studies and case reports/series were conducted across the Ovid Medline, PsycInfo, EMBASE and Cochrane Library databases. English-language articles published between 1980 and February 2019 were considered for eligibility. Included were those that objectively measured CF in individuals with neurological disease/disorder/ dysfunction between the ages of 18 and 65 years. Studies were reviewed using a modified Cochrane Data Extraction Template. Risk of bias was assessed using the Cochrane Risk of Bias tool. The review process was facilitated using Covidence software (www.covidence.org). Two authors reviewed articles independently, with a third resolving conflicts regarding article inclusion.

Results: The search identified 450 records. After duplicates were removed and remaining titles/ abstracts were screened for eligibility, 28 fulltext articles were assessed, and two studies were included in the qualitative synthesis. Studies were a priori divided into those with pharmacological, procedural or behavioural interventions. Two studies met eligibility criteria; both of these included participants with multiple sclerosis. One study utilized a procedural intervention (i.e. transcranial direct current stimulation), while the other utilized a pharmacological intervention (i.e. fampridineSR). Studies were evaluated for risk of bias, and evidence from both eligible studies was discussed. 
Conclusion: Despite the positive results of the procedural intervention, the paucity of eligible studies and the nascent nature of the field suggests that more studies are required before firm conclusions can be drawn regarding the amenability of CF to treatment.

Trial registration: The review was registered with PROSPERO (CRD42019118706).

Keywords: Cognitive fatigability; Cognitive fatigue; Mental exhaustion; Mental fatigue; Multiple sclerosis; Nervous system diseases; Stroke; Systematic review; Time-on-task; Traumatic brain injury

\section{INTRODUCTION}

Although fatigue is a well-studied concept in neurological disease, cognitive fatigability (CF) is less understood. While most studies measure fatigue using subjective self-report, fewer have measured CF objectively. Given the negative impact of CF on quality-of-life, interventions are beginning to be introduced to address this concern, but the field is in its infancy and there is little agreement on treatment approach. There is a need for a thorough review of the literature to classify the current state of the field and to identify needs moving forward.

What is meant by the term fatigue varies throughout the literature. Definitions include a state of reduced capacity for work following a period of mental or physical activity [1], a feeling of physical tiredness and lack of energy that is distinct from sadness or weakness [2], extreme tiredness with the feeling that one needs to rest [3], a feeling of lack of energy, weariness and aversion to effort [4], among other definitions [5]. Fatigue has largely been regarded as a subjective experience unique to the individual, and thus it is typically measured by self-report. A number of questionnaires have been developed to that end, with some attempting to quantify fatigue and others evaluating the impact of fatigue on daily functioning. These include: the Fatigue Severity Scale [6], the Fatigue Impact Scale [7] (and the modified version [8]), the Neurological Fatigue Index [9], the Mental Fatigue Scale [10], the Fatigue Assessment Scale
[11], the Multidimensional Fatigue Inventory [12] and the Fatigue Scale for Motor and Cognitive Functions [13, 14].

Fatigue is a common symptom in a variety of neurological diseases. It is the most commonly reported complaint in those with multiple sclerosis (MS), occurring in up to $90 \%$ of individuals affected with this disease $[15,16]$, with negative impacts on quality-of-life [17], self-esteem [18] and employability $[19,20]$. It is present in $50 \%$ of those with traumatic brain injury (TBI) [21], and affected individuals often endorse fatigue as one of the most challenging and distressing long-lasting symptoms [22, 23]. Fatigue provides a unique contribution to TBIrelated disability after controlling for injury severity, executive functions and depression [24]. It occurs in $25-85 \%$ of stroke survivors [25] and has a significant impact on the employability of the individual and society as a whole given the economic impacts [26]. A consensus report from UK stroke survivors, caregivers and health professionals noted that fatigue was in the top ten research priorities due to its debilitating consequences and the incomplete understanding of the mechanisms and treatments [27]. Negative impacts of fatigue have also been documented in those with Parkinson's disease [28, 29], amyotropic lateral sclerosis [30] and primary brain tumour [31].

Given the disparate manner in which fatigue has been addressed in the literature, a unified taxonomy has been posited that distinguishes fatigue (i.e. an individual's subjective sensations) from fatigability (i.e. objective changes in performance) in order to provide clarification and consistency in both clinical and research applications [32, 33]. While there is a body of research that focuses on physical or motor fatigability [34], the focus of the present review is CF which can be operationally defined as an inability to maintain optimal task performance throughout the duration of a sustained cognitive task $[1,35,36]$, or as a decline over time in alerting, orienting and executive control [37]. An increase in response time variability has been demonstrated to be perhaps an even more sensitive marker of CF [38]. The fact that CF can be measured objectively has implications for the lives of those affected by this condition. CF 
can often be the limiting factor to employability, but affected individuals may have difficulty accessing disability benefits given that fatigue has previously been difficult to quantify or prove to an external observer. Objective measurement allows for quantification of fatigue and thus may facilitate access to benefits.

Despite the nascent nature of the field, CF seems to have elicited more attention in the MS literature than in the literature on other neurological illnesses, with evidence of a breakdown in task performance across a variety of different cognitive tasks. Those with MS become more cognitively fatigued than healthy controls when performing information processing speed tasks, as reflected by a breakdown in their task accuracy $[35,36,39]$. Similarly, reaction time on simple sustained attention tasks increases over time in those with MS compared to controls [40-42]. Cognitive control (i.e. the combination of processes that allow adaptation of information processing depending on task goals) also declines over time in those with MS [43]. Finally, visual processing capacity has also been used as a marker of CF in those with MS [44]. Estimates of the frequency of CF is similar to those of cognitive impairment in general in individuals with MS [45], with approximately one-half of MS patients meeting defined criteria for CF in two studies $[46,47]$. The literature on $\mathrm{CF}$ in other neurological illnesses is comparatively sparse. Accelerated CF compared to healthy controls has been demonstrated in Parkinson's disease using the Attention Network Test [48], in myasthenia gravis, as evidenced by a decline in performance on repeat testing using the d2-R test [49], and in mild TBI using both attention and executive measures [50], but these studies seems to be the first to address this issue in these populations.

Mechanisms of CF have been proposed, although these studies vary with regard to whether CF is objectively or subjectively measured. CF has been associated with slower motor speed, processing speed, gender (females were found to be more susceptible, but the effect attenuated once anxiety, depression and selfefficacy were accounted for) and intelligence [47]. Sleep quality, and to a lesser extent depression, have also been found to be predictors of CF in the MS population [51], whereas others found that the relationship between CF and sleep quality diminishes when depression is controlled for [52]. It has been postulated that impaired slow wave sleep may be causing the reduced sleep quality contributing to cognitive fatigue, and so treatments targeted to improve non-REM efficiency may be warranted [53]. While sleep quality has also been found to be associated with cognitive fatigue in TBI [54], CF was not measured objectively. Nonetheless, there seems to be some converging evidence that sleep quality is likely contributory. CF can also be considered to be a reflection of brain-derived central fatigue $[55,56]$, akin to the concept of primary fatigue in MS given that it is directly related to the disease process [57]. Disruptions in circuits involving the basal ganglia, frontal cortex and thalamus have been implicated [55], and lesions in the pathways associated with arousal and attention, reticular and limbic systems and basal ganglia have been consistently demonstrated to be associated with CF [56]. Decreased white matter microstructural integrity in the striato-thalmo-cortical tracts have been demonstrated to lead to greater levels of CF in those with TBI [58], and there is a trend towards CF with reduced thickness of the pre-central gyrus, the parietal inferior supra-marginal gyrus and the opercular part of the inferior frontal gyrus in those with progressive MS [59]. Biomarkers of CF have also been identified in those with MS via functional neuroimaging, with differences in activation patterns in the attention network noted between those with MS and healthy controls before, during and after a cognitively fatiguing task [42], differences in right middle frontal gyrus recruitment in those with mild TBI [60] and connectivity differences between those who subjectively report fatigue and those who do not [61]. In addition to the influence of structural disease pathology on fatigue, there are other biological variables, such as inflammation, that impact brain-derived central fatigue as well. Indeed, the influence of pro-inflammatory cytokines on fatigue has been demonstrated in stroke [62] and MS [63]. Those with MS who subjectively report higher levels of fatigue show higher 
levels of tumour necrosis factor alpha (TNF $\alpha$ ). The levels of TNF $\alpha$ have also been found to correlate with daytime sleepiness, leading the authors of the study to suggest a cytokine-mediated fatigue syndrome in MS [63]. Similar postulations have been made in those with stroke [62]. This suggests that anti-inflammatory treatments could potentially have a secondary effect on fatigue. Given the known effects of inflammation on neurodegeneration, such treatments could potentially have disease modifying effects as well. Deficiencies in multiple neurotransmitter systems, have also been postulated to play a role in some neurological conditions, such as cholinergic and noradrenergic systems in Parkinson's [64] and dopamine in MS [65].

Potential mechanisms for CF have also been proposed based on studies of healthy individuals who have not been affected by neurological conditions. For example, high-density electroencephalography (EEG) studies have shown that compensation via recruitment of anterior frontal areas plays a role in staving off the effects of CF in healthy individuals [66]. Indeed, when performing a lengthy cognitively fatiguing task, participants in that study recruited anterior frontal regions that were not part of the primary task-related activity at baseline, thus suggesting the recruitment of additional areas specifically to compensate for fatigue-induced impairments [66]. This finding was replicated in a later study, but only in young adults [67]. The young adult group demonstrated a decline in performance over time on a sustained Stroop task that was consistent with CF. Event-related potentials in the occipital-temporal areas decreased with increased time-on-task, but event-related potentials in the central-frontal regions increased with increased time-on-task. Thus, impairments in neural processing in posterior regions were accompanied by the recruitment of frontal regions to compensate. The older adult group demonstrated the neural impairment, but there was no accompanying compensation [67]. This result suggests that in healthy individuals, the capacity to compensate for performance decrements over time declines with age. As in the neurological literature, exploration into the underlying pathological mechanisms of CF in healthy individuals is in its infancy.

There is an extensive body of literature addressing the treatment for subjectively evaluated fatigue in neurological populations. Briefly, these interventions generally stem from three different treatment approaches: pharmacological, behavioural and procedural. Pharmacological treatments are often in the form of stimulants, such as methylphenidate. The treatment is presumed to be effective given that it helps to overcome attentional difficulties and slowed processing speed, possibly due to its role as a dopamine agonist $[54,65]$. The results of studies evaluating the benefits of methylphenidate in treating subjective cognitive fatigue in TBI [68] and Parkinson's disease [69] have been positive. Other pharmacological treatments that are designed to promote wakefulness (i.e. modafinil) or improve latency to sleep (i.e. melatonin) have yet to demonstrate efficacy at addressing subjective cognitive fatigue in TBI [54]. Nonetheless, amantadine (antiviral), pemoline (stimulant) and modafinil have demonstrated positive results in treating subjective fatigue in those with MS [1].

Behavioural approaches to treating fatigue have included a variety of methods, including psychotherapy (i.e. cognitive behavioural therapy [CBT] or mindfulness), education/symptom management or cognitive rehabilitation [54]. CBT for insomnia (CBT-I) administered to those with TBI has shown some efficacy in improving sleep and decreasing fatigue [70]. A systematic review and meta-analysis on the utility of mindfulness training in the treatment of fatigue after stroke, TBI and MS concluded that these techniques were moderately effective [71]. Another systematic review evaluating the efficacy of patient education programs at improving MS-related fatigue found that these programs had a positive effect, but the authors stressed the need for multidimensional approaches given that fatigue itself is a multidimensional symptom [72]. Evidence, though preliminary, suggests that face-to-face interventions are more effective at decreasing fatigue than an on-line program in those with neurological conditions [73]. Exercise has also been explored as a potentially effective treatment 
approach, and preliminary research in MS suggests that this approach deserves further attention [74]. A meta-analysis of exercise, education and medication treatment interventions for fatigue noted that rehabilitation interventions appeared to have stronger and more significant effects than medication interventions given the ability of the former to assist people with MS in coping with their existing disabilities [75].

Procedural interventions include techniques such as light therapy, biofeedback and neuromodulation [54]. A randomized control trial (RCT) of short wavelength (blue) light therapy compared with yellow light therapy for the treatment of post-traumatic fatigue demonstrated some benefit of the blue light therapy on fatigue and daytime sleepiness, but no effect was demonstrated on performance of a task of psychomotor vigilance, raising the question of the applicability of this technique to objectively measured CF [76]. A study of EEG biofeedback over 25 sessions noted a benefit in the TBI treatment group in terms of both general and mental fatigue [77], but the study was considered to be underpowered [78]. A recent review of the use of transcranial magnetic stimulation in MS found preliminary evidence of a beneficial impact on fatigue, but replication in welldesigned RCTs was recommended [79]. A similar review in the stroke literature did not yield any studies evaluating this technique for treatment of fatigue, emphasizing that this is an emerging technology with the need for further study [80].

A systematic review of interventions for fatigue following TBI concluded that evidence was insufficient to recommend or contradict particular treatments and called for high-quality research that incorporates appropriate definitions and measurement of fatigue [78]. This seems to be the case for fatigue interventions in general as it relates to neurological illness and is the impetus for pursuing the current review. There is a clear paucity of research to address the treatment effects of objectively measured CF. It is this gap in the literature that led to the current project. The aim of this systematic review is thus to determine which procedural, behavioural and pharmacological treatments for objectively measured $\mathrm{CF}$ are available to people living with neurological conditions. The presence of subjectively measured cognitive fatigue has been demonstrated to be predictive of MS disease progression in the form of relapses and brain atrophy [81]. As such, early detection of fatigue, and the treatment of such, may have implications for disease course. If we can ameliorate $\mathrm{CF}$, then perhaps we can positively influence not only quality-of-life, but also longterm disease outcomes.

\section{METHODS}

\section{Compliance with Ethics Guidelines}

This article is based on previously conducted studies and does not contain any studies with human participants or animals performed by any of the authors.

\section{Protocol and Registration}

The methods and criteria for study inclusion as discussed below were outlined prospectively. The protocol document was registered with PROSPERO, an international database of prospectively registered systematic reviews (http://www.crd.york.ac.uk/PROSPERO/display_ record.php?ID=CRD42019118706). Although there were other protocols listed on PROSPERO that evaluated fatigue, there were no other protocols focusing on objectively measured CF. As such, the uniqueness of the current project was assured.

\section{Study Eligibility Criteria and Information Sources}

In accordance with PRISMA guidelines [82-85], systematic searches for RCTs, case-controlled studies and case reports/series were conducted across the Ovid Medline, PsycInfo, EMBASE and Cochrane Library databases. Grey literature (abstracts, conference proceedings, editorials) and existing reviews (narrative, scoping, systematic reviews) were excluded. The search strategies were established with the assistance of an experienced healthcare librarian. An 
Table 1 Search strategy for Ovid Medline

\begin{tabular}{llrl}
\hline Number & Searches & Results & Type \\
\hline 1 & Mental fatigue.mp. or mental fatigue/ & 2064 & Advanced \\
2 & ((Mental or cognitive) adj fatigue).tw. & 1191 & Advanced \\
3 & Fatigability.mp. & 2122 & Advanced \\
4 & Time on task.mp. & 555 & Advanced \\
5 & Mental exhaustion.mp. & 106 & Advanced \\
6 & 1 or 2 or 3 or 4 or 5 & 4951 & Advanced \\
7 & Multiple sclerosis.mp. or multiple sclerosis/ & 76,134 & Advanced \\
8 & Traumatic brain injury.mp. or brain injuries, traumatic/ & 32,086 & Advanced \\
9 & Stroke.mp. or STROKE/ & 261,031 & Advanced \\
10 & Parkinson disease.mp. or Parkinson disease/ & 68,219 & Advanced \\
11 & Nervous system.mp. or nervous system/ & 467,290 & Advanced \\
12 & 7 or 8 or 9 or 10 or 11 & 871,320 & Advanced \\
13 & 6 or 12 & 550 & Advanced \\
14 & Treatment.mp. & $4,429,717$ & Advanced \\
15 & Intervention.mp. & 540,513 & Advanced \\
16 & 14 or 15 & $4,763,175$ & Advanced \\
17 & 13 and 16 & 139 & Advanced \\
18 & Limit 17 to yr $=$ "1980 - current" & 133 & Advanced \\
\hline
\end{tabular}

example search strategy for Medline is provided in Table 1, and the complete search strategies for all databases are provided in the supplementary materials (Electronic Supplementary Material [ESM] S1-S4). English-language articles published between 1 January 1980 and February 2019 were considered for eligibility. The date last searched was 12 February 2019. Supplemental approaches to identify eligible studies were also utilized and included checking reference lists and searching trial registries.

Studies evaluating behavioural, procedural and/or pharmacological interventions were included in this review. The target of the interventions must have been treatment and/or amelioration of CF as the primary or secondary outcome. Studies with interventions targeting other primary, secondary and tertiary outcomes (excluding subjective fatigue [i.e. self-report], cognition, quality-of-life, mood and sleep, etc.) were included as long as the treatment of CF remained the primary or secondary objective. Study participants were individuals with neurological disease/disorder/dysfunction between the ages of 18 and 65 years.

The review process was facilitated using Covidence software (www.covidence.org). Following the database searches duplicates were removed. Two reviewers (JB, ALB) then independently screened titles and abstracts of the retrieved records. A third blinded reviewer (LW) resolved conflicts. Two reviewers (JB, ALB) then independently assessed full-text articles for eligibility using a modified Cochrane Data Extraction Template. A third blinded reviewer (LW) resolved any conflicts. Following data extraction, retained articles underwent qualitative synthesis by all three authors. The study 
selection process is shown in a PRISMA flow diagram (Fig. 1).

\section{Data Extraction}

Data were extracted using a modified Cochrane Data Extraction Template (ESM S5). Reviewers were trained together in how to extract data using the template. The data extraction sheet was pilot tested on three articles and refined accordingly. Following extraction of the data from the eligible papers, information was placed in a table. Information was extracted according to PICOS criteria [82]: population, interventions, comparator, outcomes and study design.

\section{Risk of Bias Assessment}

Risk of bias was assessed using the Cochrane Risk of Bias assessment [86] (ESM 6). This tool assesses selection bias, performance bias, detection bias, attrition bias and reporting bias. Two reviewers (JB, ALB) completed the risk of bias assessment independently. Once results were

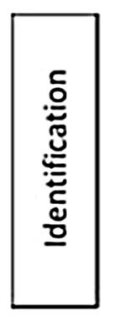

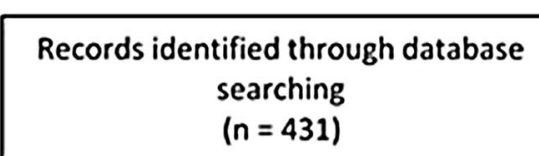

$(n=431)$
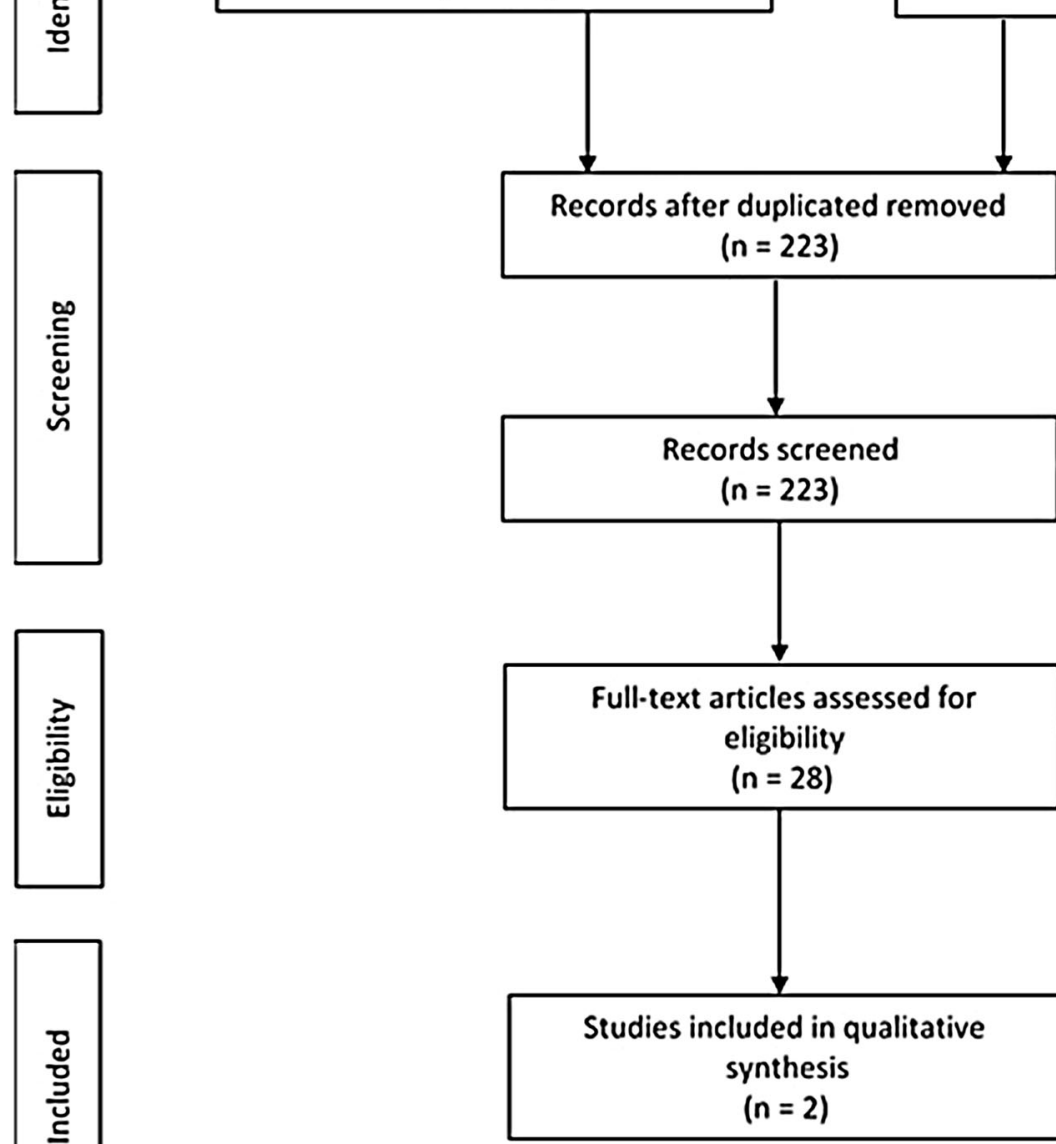

Additional records identified through other sources ( $n=19)$
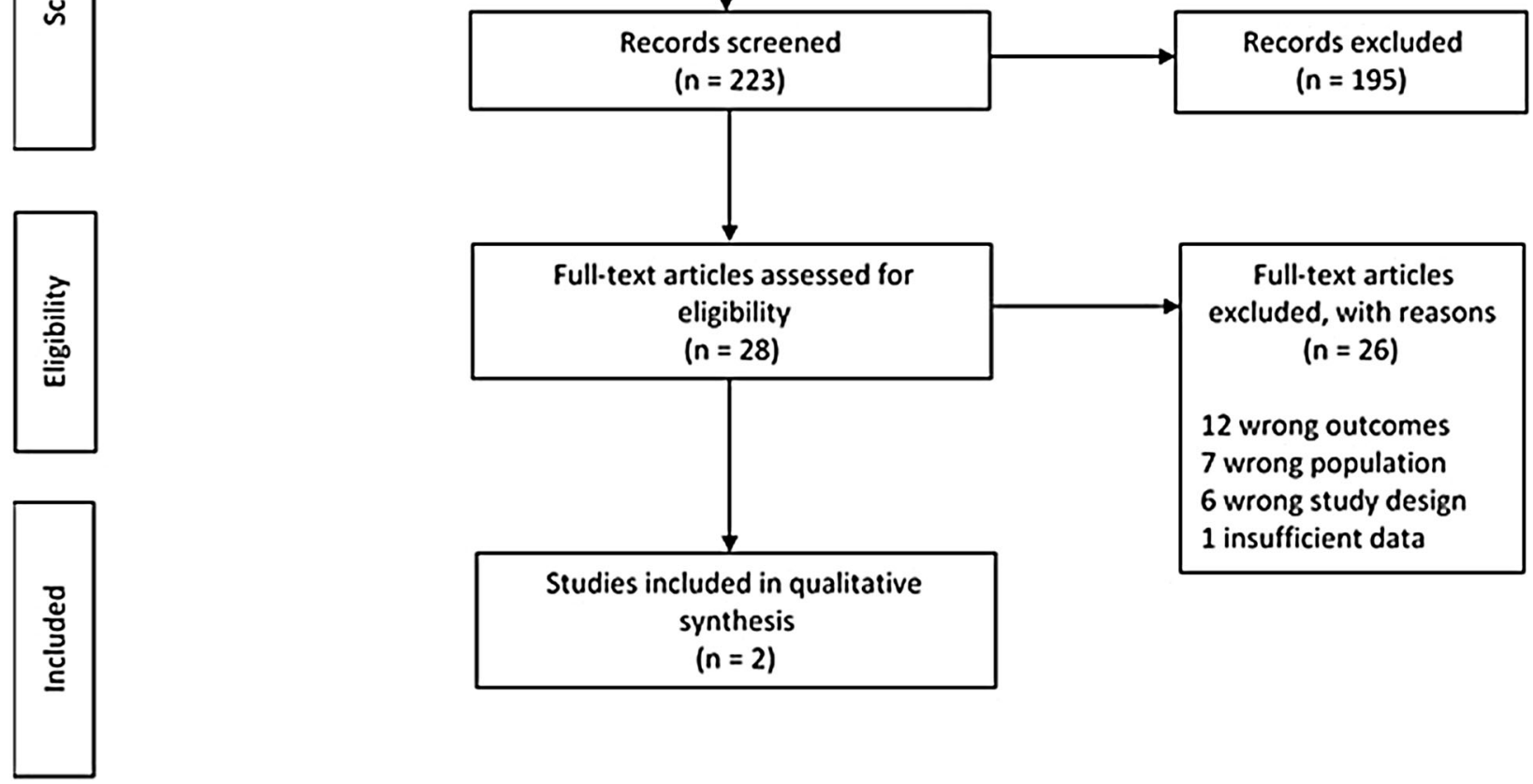

Fig. 1 PRISMA (Preferred Reporting Items for Systematic Reviews and Meta-Analyses) flow diagram 
compared, any disagreements were resolved by the third reviewer (LW).

\section{Strategy for Data Synthesis}

Given the qualitative nature of the review, main results are reported in narrative format. The review includes discussion of the various interventions used (classified as either pharmacological, behavioural or procedural interventions), as well as sample characteristics. A summary of the results of each study is provided and a narrative synthesis is discussed. Quantitative meta-analyses were not planned.

\section{Risk of Bias Across Studies}

Cumulative evidence may have been affected by publication bias and selective reporting within studies.

\section{RESULTS}

\section{Study Selection}

A total of 431 publications were identified through the database search process, with an additional 19 publications identified through other sources (e.g. reference lists). After deleting duplicates, 223 publications remained for further analysis. The abstracts of these articles were screened by two reviewers (JB, ALB), who assessed that 195 publications did not meet eligibility criteria; thus these were eliminated, leaving 28 articles for full-text screening. After reviewing each of these remaining articles, the reviewers excluded 26 of these for various reasons, including ineligible outcome (12 publications), wrong population (7), ineligible study design (6) and insufficient data (1). After this process, two publications remained that met all eligibility criteria; these were included in the qualitative synthesis.

\section{Study Characteristics}

Both studies included in the qualitative synthesis were RCTs targeted at improving objectively evaluated CF (Table 2). The Morrow et al. study [87] was a double-blind placebocontrolled cross-over design evaluating a pharmacological treatment (fampridine-SR $10 \mathrm{mg}$ twice daily). The intervention included 4 weeks of either fampridine-SR or placebo, a 1-week wash-out period and an additional 4 weeks of either fampridine-SR or placebo. This was a single-center pilot study that took place within the MS Clinic of London Health Sciences Centre in London, Ontario, Canada.

The Fiene et al. study [88] was a randomized, single-blind, within-subject, sham-controlled RCT investigating the effects of anodal transcranial direct current stimulation (tDCS) over the left dorsolateral prefrontal cortex (DLPFC) on objective performance-based measures of $\mathrm{CF}$ in MS during prolonged testing. This procedural interventional study was conducted at a single center at the University of Magdeburg Hospital in Magdeburg, Germany.

\section{Participants}

Notably, both eligible studies were conducted with participants with MS. No studies with participants with other neurological conditions were eligible, although a number of reviewed studies were conducted including those with stroke or TBI.

The Morrow et al. study [87] enrolled 60 individuals with a confirmed diagnosis of MS. Subtypes of MS included in the study were relapsing-remitting (41 participants), secondary-progressive (14) and primary progressive (5). Participants were randomized to either the early treatment or late treatment group. Groups were matched on age, education, years since diagnosis, sex, ethnicity, use of disease-modifying medications and MS type (see Table 2). Approximately three-quarters of participants were female, as is typical for most studies of MS given the sex distribution; most had at least a high school education; years since diagnosis was approximately 10; mean age was approximately 46 years; $>90 \%$ were Caucasian; disability rating on the Expanded Disability Status Score (EDSS) [89] was approximately 3-3.5; and 


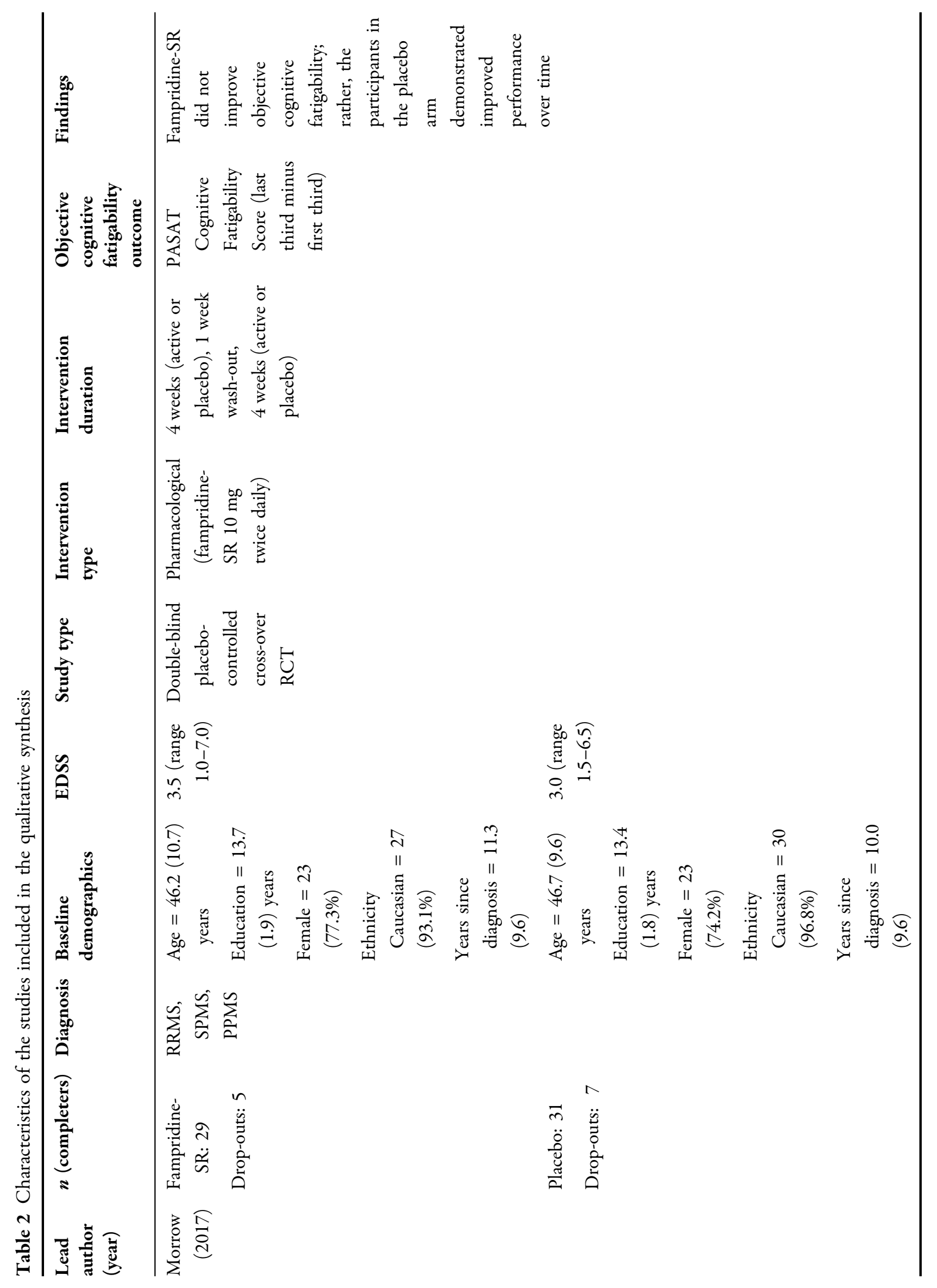


260

Neurol Ther (2019) 8:251-271

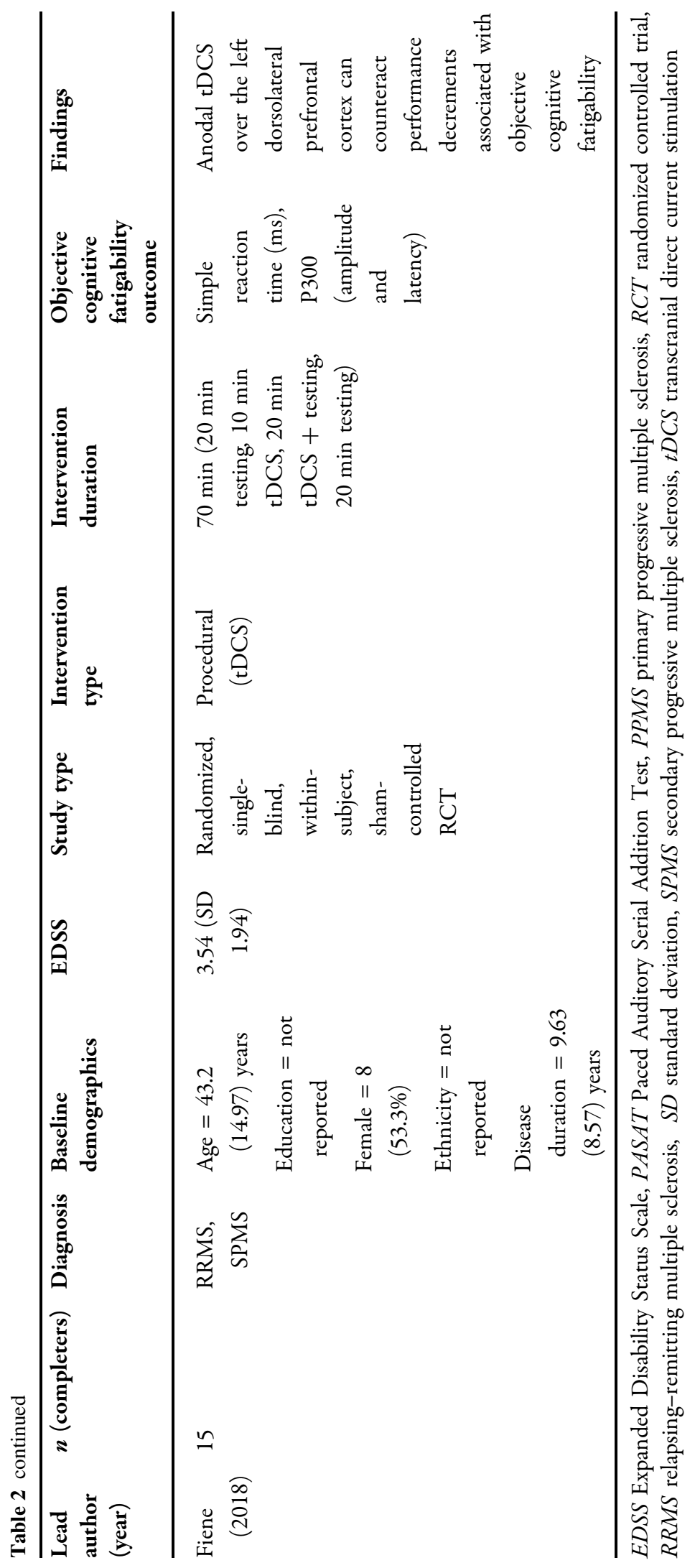

$\triangle$ Adis 
approximately one-half were being treated with disease-modifying medication.

The Fiene et al. study [88] enrolled 15 individuals with a confirmed diagnosis of MS, of whom 14 had relapsing-remitting MS, and one had secondary-progressive MS. Participants received either anodal or sham tDCS in a randomized, counterbalanced order. Proportionally, there were more males in this study than in the Morrow et al. study [87] (8 females; 7 males), with an almost 1:1 ratio. Mean age of participants was approximately 43 years. Education and ethnicity were not reported. Disease duration was approximately 10 years, and disability rating on the EDSS was approximately 3.5.

Overall, the participants between the two studies were largely comparable, with the exception of a greater proportion of males and a lower proportion of participants with progressive MS in the Fiene et al. study [88]. Notably, numbers are small even across both studies combined.

\section{Interventions}

The Morrow et al. study [87] utilized a pharmacological intervention in the form of fampridine-SR (4-aminopyridine), a selective neuronal potassium-channel blocker. This is a slow-release oral medication that has been designed to treat fatigue. Studies have demonstrated improvement in ambulation, subjective fatigue and endurance [90-93]. The hypothesized mode of action is improvement of nerve conduction via enhanced action potential formation $[94,95]$, which in turn is expected to improve CF by improving nerve conduction. The packaging and capsules of fampridine-SR and placebo were identical, and only the pharmacist was unblinded. Participants were treated with either substance for 4 weeks in block 1 . There was a minimum of 1-week washout period, and then they entered block 2 and received the opposite substance to what they received in block 1 for an additional 4 weeks. Outcomes were assessed at the beginning and end of both block 1 and 2, for a total of four evaluations.

The Fiene et al. study [88] utilized a procedural intervention in the form of tDCS. This is a non-invasive technique to modulate cortical excitability in targeted brain regions. The polarity of the current determines whether the response is cortical excitation via depolarization of neuronal membranes (anodal tDCS) or a decrease in cortical reactivity via hyperpolarization (cathodal tDCS). While this was the first study to evaluate the impact of tDCS on objectively measured $\mathrm{CF}$, the rationale for its use came from the positive outcomes of anodal tDCS on subjective fatigue in other studies [96-99]. Participants underwent the protocol twice, once using anodal stimulation and once using sham stimulation. These sessions were separated by at least 1 week. Each treatment session consisted of three testing blocks, preceded by a short practice trial. The first block was approximately $20 \mathrm{~min}$ long and was a baseline control condition without tDCS. Electrodes were then attached to the scalps of participants during a 5-min break. During the second block participants received $10 \mathrm{~min}$ of tDCS pre-stimulation to ensure stable stimulation effects. This was followed by approximately 20 min of tDCS during which participants were assessed (see Outcomes section for outcomes). After another 5-min break, the third testing block consisted of the measurement of poststimulation effects. The two treatment sessions were separated by a minimum of 7 days, and each session took place at about the same time of day.

\section{Outcomes}

The Morrow et al. study [87] operationally defined cognitive fatigue according to the definition proposed by Schwid et al. [100] which states that cognitive fatigue is a decline in cognitive performance during a task requiring sustained cognitive activity (meeting our current definition of $\mathrm{CF}$ ). Their results demonstrated that the participants with MS in the placebo arm showed more benefit, both in terms of raw Paced Auditory Serial Addition Test (PASAT) scores and the CF variable. Thus, in contrast to the hypothesized outcome, the participants in the placebo arm showed less vulnerability to CF at follow-up than those who had received active 
treatment with fampridine-SR. The authors noted some limitations to their study. The study was small and administered at only one site, perhaps resulting in the study being underpowered. They also postulated that perhaps only some of the participants were responders to the medication given that this has been demonstrated in another study evaluating timed walk performance [101]. Additionally, they noted that they did not identify possible responders based on self-reported fatigue, nor did they conduct a full cognitive assessment at baseline. Lastly, they noted that there was a practice effect on the PASAT that led to many participants not meeting criteria for CF when entering block 2 .

The Fiene et al. study [88] operationally defined objective cognitive fatigue (meeting our current definition of $\mathrm{CF}$ ) as the failure to sustain effort over the course of a testing session, as quantified by changes in simple reaction time (RT) and P300 amplitude components with time-on-task. Their results demonstrated that after a single dose of $<30$ min of tDCS-induced modulation of the frontal cortex there were changes in both objective cognitive performance and neurophysiological markers. Participants with MS undergoing the sham procedure showed the expected increase in RT over the course of the cognitively fatiguing task, which was interpreted as objective evidence of CF. Participants with MS who received tDCS did not show any change in RT over time, suggesting that neuromodulation of the left DLPFC may allow those with MS to maintain their performance over time and overcome their vulnerability to CF. Neurophysiological evidence of this effect was demonstrated by the increase in P300 amplitude score during tDCS stimulation which persisted after the end of the treatment. The authors interpreted these findings as demonstrating evidence that the left DLPFC is functionally relevant in objective CF.

\section{Risk of Bias in the Individual Studies}

Table 3 provides additional information on the risk of bias in the two eligible studies. The selection, detection, attrition, performance and reporting bias indices of the Morrow et al. study [87] were considered to be low. In contrast, the various indices of bias for the Fiene et al. study [88] were more variable. Specifically, the detection, attrition and reporting biases were considered to be low, but performance bias was high given that the study was a single-blind study only, such that the person delivering the tDCS treatment was not blinded to the treatment arm. Selection bias was unclear given that the authors did not specify how participants were randomized to either the sham or anodal tDCS arm.

\section{Synthesis of Results}

Of the two studies meeting the eligibility criteria, both involved individuals with MS. Studies evaluating objectively measured $\mathrm{CF}$ in other neurological conditions were few, and none met the criteria for inclusion. Taking both studies together, there was a total of 75 participants with MS who met the eligibility criteria, of whom only 44 received either of the two active treatments. The Morrow et al. study [87] included participants with both inflammatory and non-inflammatory MS (i.e. secondary progressive MS [SPMS] and primary progressive MS), whereas the Fiene et al. study [88] included only those in the inflammatory phase of the disease (i.e. relapsing-remitting MS and one participant with SPMS). Thus, conclusions about the efficacy of the procedural intervention cannot be generalized to those with noninflammatory MS. Neither study has been replicated, and conclusions must be considered to be preliminary. To summarize, although both studies can be considered as providing Class I evidence, given that they were RCTs, there is a clear need for replication of these findings before broad prescriptive statements can be made.

Although both studies objectively measured $\mathrm{CF}$, the two reviewed studies utilized differing methodologies, with one using performance decline on the PASAT and the other using both performance and neurophysiological measures. Only the procedural intervention demonstrated efficacy (both performance-based and 
Table 3 Risk of bias in the two eligible studies

\begin{tabular}{lll}
\hline Type of bias & Morrow et al. (2017) [87] & Fiene et al. (2018) [88] \\
\hline Random sequence generation (selection bias) & Low & Unclear \\
Allocation concealment (selection bias) & Low & Unclear \\
Baseline outcome similarity (detection bias) & Low & Low \\
Incomplete outcome data (attrition bias) & Low & Low \\
Blinding of participants and personnel (performance bias) & Low & High \\
Selective reporting (reporting bias) & Low & Low \\
Other bias & Low & Low \\
\hline
\end{tabular}

Low low risk of bias, High high risk of bias, unclear uncertain risk of bias

neurophysiological). In addition to the objective findings of $\mathrm{CF}$, both group of authors reported an increase in perceived or subjective fatigue during the testing sessions [88]. Despite subjective fatigue being present, objective metrics did not show a performance decrement in the treatment group. Findings thus remain consistent with past literature in demonstrating a discrepancy between participant perceptions and their objective performance $[102,103]$.

\section{DISCUSSION}

The aim of this systematic review was to determine which procedural, behavioural and pharmacological treatments for objectively measured $\mathrm{CF}$ are available to people living with neurological conditions. Only two studies met our prospective eligibility criteria; both published within the last 2 years. This paucity of published studies suggests that the study of objectively measured CF is in its infancy and much work is yet to be done. Although studies initially reviewed were those that enrolled participants with a variety of neurological conditions, the two studies that met all eligibility criteria were restricted to those involving persons living with MS. Given that fatigue is such a prominent aspect of a wide variety of neurological conditions, the eligibility of only these two studies in the present review suggests that MS researchers are leading the field in this area.
Of the two eligible studies, one utilized a pharmacological intervention (i.e. rampridineSR) [87] and the other a procedural one (i.e. tDCS) [88]. Only the latter demonstrated efficacy with regard to the treatment of objectively measured CF. Specifically, tDCS to the left DLPFC appeared to prevent the increase in RT typically demonstrated in those with MS after a cognitively fatiguing task. Thus, the expected performance decrements as a result of sustained cognitive effort were prevented. The study also demonstrated neurophysiological evidence of the treatment effect given the increased P300 amplitude score that persisted after the end of the treatment. The importance of the left DLPFC in regulating CF is consistent with our own work which has demonstrated that dysfunction in cerebral blood flow allocation to the middle frontal gyrus toward the end of a cognitively fatiguing task seems to underlie the CF effects observed in those with MS. Specifically, those with MS seem unable to recruit the middle frontal gyrus during periods of high cognitive workload to circumvent the performance decline over time [42]. The Fiene et al. study [88] demonstrates that these processes can potentially be manipulated via tDCS, such that the brains of those with MS can possibly be functionally altered to reduce the inherent vulnerability to CF. This finding is promising, yet clearly preliminary and in need of replication. Similarly, the study was prone to performance bias (with unclear selection bias), and 
thus conclusions must be further tempered. Given that the effect of treatment was in a single test session only, it is unclear if the beneficial effect of tDCS on CF is temporary, or if effects may be more long-lasting. A focused review of studies utilizing repeated anodal tDCS treatments (up to 6 weeks) to treat subjective fatigue in MS reported stable improvements up to 3 weeks following the last treatment session [104], thus future work should focus on whether a series of treatments over time can lead to similar persistent changes in regard to objectively measured CF.

The lack of positive outcomes in the pharmacological study reviewed [87] does not necessarily preclude the potential benefits of this or other medication options in the treatment of fatigue. At this time, however, there is insufficient evidence to make any claims in either direction given the lack of research. Pharmacological interventions have been effective in the treatment of subjective fatigue in a variety of conditions $[1,68,69]$, and thus further study of the effects of medication on objectively measured CF is warranted, particularly given the evidence for potential biomarker targets [62-65]. The same holds true for behavioural interventions. Although no studies have yet addressed the impact of behavioural interventions on objective CF specifically, the positive impact of these interventions on subjective fatigue $[71,72,75]$ can provide some direction in terms of potentially fruitful options to pursue. Given the relationship between CF, sleep quality [53, 54, 105] and mood [52], a behavioural intervention designed to target sleep quality and mood, such as CBT, or more specifically CBT-I [106], may be warranted. However, as previously suggested, one must consider the multidimensional nature of fatigue when planning and designing treatment interventions, and thus a multi-faceted approach targeting lifestyle factors and coping techniques (e.g. fatigue management education supplemented by elements of CBT-I and exercise) might have a greater chance of efficacy [72]. If such behavioural techniques are effective, the potential additive effects of a combined behavioural and procedural approach could then be explored.
Not only does this review highlight the lack of clarity with regard to treatments to target CF, as related to the original aim, but it also highlights that there is no clear consensus regarding how CF is measured. Although studies in this area seem to agree that a performance decrement over time is indicative of $\mathrm{CF}$, how this decrement is quantified varies. A number of authors have utilized the PASAT to quantify CF by evaluating performance accuracy toward the end of the task as compared to the beginning $[36,39,107]$, but studies vary with regard to whether to compare the first half performance with second half, or the first third with last third. A ratio of accurate performance on a variety of 60-s tasks evaluating response inhibition, processing speed and working memory compared to equivalent 180-s tasks has also been utilized as a marker, with some evidence that the CF effect may be domain specific [46]. Others have utilized speeded tasks to demonstrate that response time increases over time, but even within this method, individual tasks vary. Some have utilized a psychomotor vigilance task and compared performance between the first and last quintiles (i.e. across task) [42], while others have utilized computerized test battery for attention performance and compared performance between administrations before and after either cognitively or motorically fatiguing tasks (i.e. across session) [40]. Changes in response time variability has been shown to be an even more sensitive marker than response time changes over time, perhaps representing attentional lapses or other breakdowns in cognitive control [38]; however, these changes have been evaluated only in healthy controls, and the methodology has not yet been applied in neurological samples as a measure of CF. Response time variability has been demonstrated to correlate with subjectively reported fatigue in an MS sample [108] and thus could easily be applied as a measure of objective CF. Although other methods have also been utilized, the common theme is tasks that require sustained attention and/or effort over time $[41,43,44]$.

The Fiene et al. study [88] also highlighted that in addition to performance metrics, as discussed thus far, there are biomarkers that can 
also be incorporated into the study of CF; for example, the authors demonstrated that lower amplitudes and prolonged latencies of the P300 event-related potential are indicative of CF. The utility of this electrophysiological marker had previously been demonstrated by others [46] and has also shown some correlation with other clinical measures. Functional neuroimaging is also a promising avenue of research with respect to biomarkers. Our own work using arterial spin labeling functional magnetic resonance imaging has demonstrated differences in frontoparietal attentional network activation before, during and after a cognitively fatiguing task between those with MS and healthy controls [42]. Those with MS engaged the middle frontal gyrus more than the healthy controls during the cognitive task as a whole. However, the controls showed greater activation of this area only during the last quintile of the task. Thus, the performance decline in the MS group seemed to be due to their inability to recruit the middle frontal gyrus at the end of the task. Others have utilized resting-state functional connectivity to demonstrate alterations in the connectivity between the insula and posterior cingulate gyrus, and also between the right thalamus and right precentral gyrus, between fatigued and non-fatigued individuals with MS [61]. These authors suggested that disruption in sensorimotor, high-order motor and non-motor executive function likely contributes to the neural mechanism of fatigue in MS. Although the influence of secondary factors (e.g. diseaserelated symptoms such as mood) must also be considered, these studies provide further evidence for primary (i.e. central) fatigue and clearly elucidate potential targets of neuromodulatory techniques.

As previously stated, the study of fatigue in neurological conditions is fraught with varying definitions and methodology that highlight the need for a clear conceptual framework from which to guide further study. Harrison et al. [109] summarized the available evidence for measures of CF in MS and made the following recommendations. First, they called for the development of a guiding theory of $\mathrm{CF}$ that considers differences between primary and secondary fatigue and broader psychosocial models. This point aligns with recommendations by Kluger et al. recommending a unified taxonomy for studying fatigue and fatigability in neurological illness [32]. Second, they suggested that future research examine the ecological and construct validity of existing assessments. Third, they suggested that research explore whether the more promising CF measures are correlated with impaired functioning after accounting for possible confounds. Fourth, they highlighted the need for outcome assessments to be able to identify clinically meaningful improvement [109]. A last point made by Harrison et al. that we wish to echo here is that the quest for improving the objective measurement of CF does not imply that subjectively measured fatigue is somehow less relevant or important [109]. Clearly, patient perceptions of their own experience should be guiding both clinicians in terms of helping them manage their symptoms, and researchers by ensuring that research questions address concerns that are relevant to the patient populations under study. Improving the quality-of-life of those affected by neurological conditions should be our guiding principle. In addition to these astute recommendations, we would like to add two further points. First, that current and emerging biomarkers of CF be incorporated into the study of this phenomenon (including their relationship to performance indicators) and second, that research moving forward focusses on establishing effective interventions to target CF. Preliminary evidence suggests that a variety of approaches show promise, but there is a need for well-designed RCTs that incorporate the recommendations noted above in their trial design. Thorough evaluation of behavioural, procedural and pharmacological interventions individually and then in combination is needed.

There are some limitations associated with the current review. First, the studies meeting the inclusion criteria were limited to those involving participants affected by MS. This highlights the need for further study in other neurological conditions and the lack of understanding in the literature about whether CF manifests differently (or responds differently to treatment) depending upon the underlying 
pathological mechanism. Second, the studies included were conducted using small sample sizes, which limits the generalizability of the findings. Third, one of the studies had variable or unclear bias. Fourth, systematic reviews assume that the evaluation techniques are consistent across studies, but this may not necessarily be true [110]. Fifth, the eligibility criteria for inclusion was quite strict and so relaxing these criteria may have resulted in more eligible studies. For example, expanding the age range beyond 65 years may have yielded studies conducted in those with neurodegenerative dementias (although significant cognitive impairment might be considered a confounding factor). Finally, grey literature (i.e. abstracts, conference proceedings) was not reviewed; this, and the fact that negative trials are often not reported, may have resulted in a publication bias or a "file-drawer effect" [110].

\section{CONCLUSION}

Although in this review, the only eligible study to demonstrate efficacy in ameliorating CF was a procedural one (i.e. tDCS), it cannot be concluded that procedural interventions are superior to either behavioural or pharmacological ones given the lack of study in this area. Similarly, one cannot conclude that pharmacological interventions are unsuccessful on the basis of only one unsuccessful trial (i.e. fampridineSR). The current findings do not lead to any clear recommendations about appropriate methods of CF measurement or associated treatment approach. There is simply insufficient information to provide clear guidance. This review highlights the considerable need for further study according to the recommendations noted above.

\section{ACKNOWLEDGEMENTS}

The authors would like to thank Dr. Giorgio Tasca for his assistance in the initial planning stages of this review. We would also like to thank librarian Risa Shorr for her assistance in finalizing a search strategy.
Funding. No funding or sponsorship was received for this study.

Authorship. All named authors meet the International Committee of Medical Journal Editors (ICMJE) criteria for authorship for this article, take responsibility for the integrity of the work as a whole, and have given their approval for this version to be published.

Disclosures. Jason Berard and Alyssa Lindsay-Brown have nothing to disclose. Lisa Walker has received research grants from Serono Canada and Bayer HealthCare: Pharmaceuticals Division, but these are unrelated to the current project.

Compliance with Ethics Guidelines. This article is based on previously conducted studies and does not contain any studies with human participants or animals performed by any of the authors.

Data Availability. Data sharing is not applicable to this article as no datasets were generated or analysed during the current study.

Open Access. This article is distributed under the terms of the Creative Commons Attribution-NonCommercial 4.0 International License (http://creativecommons.org/licenses/ by-nc/4.0/), which permits any noncommercial use, distribution, and reproduction in any medium, provided you give appropriate credit to the original author(s) and the source, provide a link to the Creative Commons license, and indicate if changes were made.

\section{REFERENCES}

1. Schwid SR, Covington M, Segal BM, Goodman AD. Fatigue in multiple sclerosis: current understanding and future directions. J Rehabil Res Dev. 2002;39:211-24.

2. Krupp L, Alvarez L, LaRocca NG, Scheinberg L. Fatigue in multiple sclerosis. Arch Neurol. 1988;45:435-7. 
3. Freal JE, Draft GH, Coryell J. Symptomatic faigue in multiple sclerosis. Arch Phys Med Rehabil. 1984;65:135-8.

4. Mead G, Lynch J, Greig C, et al. Evaluation of fatigue scales in stroke patients. Stroke. 2007;38:2090-5.

5. Staub F, Bogousslavsky J. Fatigue after stroke: a major but neglected issue. Cerebrovasc Dis Basel Switz. 2001;12:75-81.

6. Krupp LB, LaRocca NG, Muir-Nash J, Steinberg AD. The Fatigue Severity Scale: application to patients with multiple sclerosis and systemic lupus erythematosus. Arch Neurol. 1989;46:1121-3.

7. Fisk JD, Ritvo PC, Ross L, Haase DA, Marrie TJ, Schlech WF. Measuring the functional impact of fatigue: initial validation of the Fatigue Impact Scale. Clin Infect Dis. 1994;18:S79-83.

8. Amtmann D, Bamer AM, Noonan V, Lang N, Kim J, Cook KF. Comparison of the psychometric properties of two fatigue scales in multiple sclerosis. Rehabil Psychol. 2012;57:159-66.

9. Mills RJ, Young CA, Pallant JF, Tennant A. Development of a patient reported outcome scale for fatigue in multiple sclerosis: the Neurological Fatigue Index (NFI-MS). Health Qual Life Outcomes. 2010;8:22.

10. Johansson B, Starmark A, Berglund P, Rodholm M, Ronnback L. A self-assessment questionnaire for mental fatigue and related symptoms after neurological disorders and injuries. Brain Inj. 2010;24:2-12.

11. Michielsen HJ, De Vries J, Van Heck GL. Psychometric qualities of a brief self-rated fatigue measure: the Fatigue Assessment Scale. J Psychosom Res. 2003;54:345-52.

12. Smets EM, Garssen B, Bonke B, et al. The multidimensional fatigue inventory (MFI) psychometric qualities of an instrument to assess fatigue. J Psychosom Res. 1995;39:315-25.

13. Penner IK, Raselli C, Stöcklin M, Opwis K, Kappos L, Calabrese $\mathrm{P}$. The fatigue scale for motor and cognitive functions (FSMC): validation of a new instrument to assess multiple sclerosis-related fatigue. Mult Scler. 2009;15:1509-17.

14. Hubacher M, Calabrese P, Bassetti C, Carota A, Stöcklin M, Penner IK. Assessment of post-stroke fatigue: the fatigue scale for motor and cognitive functions. Eur Neurol. 2012;67:377-84.
15. Brassington JC, Marsh NV. Neuropsychological aspects of multiple sclerosis. Neuropsychol Rev. 1998;8:43-7.

16. Minden SL, Frankel D, Hadden L, Perloffp J, Srinath KP, Hoaglin DC. The Sonya Slifka Longitudinal Multiple Sclerosis Study: methods and sample characteristics. Mult Scler. 2006;12:24-38.

17. Opara JA, Jaracz K, Brola W. Quality of life in multiple sclerosis. J Med Life. 2010;3:352-8.

18. Janardhan V, Bakshi R. Quality of life in patients iwth multiple sclerosis: the impact of fatigue and depression. J Neurol Sci. 2002;205:51-8.

19. Smith MM, Arnett PA. Factors related to employment status changes in individuals with multiple sclerosis. Mult Scler. 2005;11:602-9.

20. Cadden M, Arnett PA. Factors associated with employment status in individuals with multiple sclerosis. Int J MS Care. 2015;15:284-91.

21. LaChapelle DL, Finlayson M. An evaluation of subjective and objective measures of fatigue in patients with brain injury and healthy controls. Brain Inj. 1998;12:649-59.

22. Lannsjö M, af Geijerstam JL, Johansson U, Bring J, Borg J. Prevalence and structure of symptoms at 3 months after mild traumatic brain injury in a national cohort. Brain Inj. 2009;23:213-9.

23. Cantor JB, Gordon W, Gumber S. What is post TBI fatigue? NeuroRehabilitation. 2013;32:875-83.

24. Juengst S, Skidmore E, Arenth PM, Niyonkuru C, Raina KD. The unique contribution of fatigue to disability in community dwelling adults with traumatic brain injury. Arch Phys Med Rehabil. 2013;94:74-9.

25. Cumming TB, Packer M, Kramer SF, et al. The prevalence of fatigue after stroke: a systematic review and meta-analysis. Int $\mathrm{J}$ Stroke. 2016;11:968-77.

26. Andersen G, Christensen D, Kirkevold M, et al. Poststroke fatigue and return to work: a 2 -year followup. Acta Neurol Scand. 2012;125:248-53.

27. Pollock A, St. George B, Fenton M, Firkins L. Top 10 research priorities relating to life after stroke-consensus from stroke survivors, caregivers, and health professionals. Int J Stroke. 2014;9:313-20.

28. Lou JS, Kearns G, Oken B, Sexton G, Nutt J. Exacerbated physical fatigue and mental fatigue in Parkinson's disease. Mov Disord. 2001;16:190-6. 
29. Nagy A, Schrag A. Neuropsychiatric aspects of Parkinson's disease. J Neural Transm. 2019;126(7):889-96.

30. Lou J, Reeves A, Benice T, Sexton G. Fatigue and depression are associated with poor quality of life in ALS. Neurology. 2003;60:122-3.

31. Day J, Yust-Katz S, Cachia D, et al. Interventions for the management of fatigue in adults with a primary brain tumour. Cochrane Database Syst Rev. 2016;4:CD011376. https://doi.org/10.1002/ 14651858.CD011376.pub2.

32. Kluger BP, Krupp LB, Enoka RM. Fatigue and fatigability in neurologic illnesses: proposal for a unified taxonomy. Neurology. 2013;80:409-16.

33. Kluger BM, Herlofson K, Chou KL, et al. Parkinson's disease-related fatigue: a case definition and recommendations for clinical research. Mov Disord. 2016;31:625-31.

34. Bigland-Ritchie B, Cafarelli E, Vollestad NK. Fatigue of submaximal static contractions. Acta Physiol Scand. 1986;556:137-48.

35. Bryant M, Chiaravalloti ND, DeLuca J. Objective measurement of cognitive fatigue in multiple sclerosis. Rehabil Psychol. 2004;49:114-22.

36. Walker LAS, Berard JA, Berrigan LI, Rees LM, Freedman MS. Detecting cognitive fatigue in multiple sclerosis: method matters. J Neurol Sci. 2012;316:86-92.

37. Holtzer R, Shuman M, Mahoney JR, Lipton R, Verghese J. Cognitive fatigue defined in the context of attention networks. Neuropsychol Dev Cogn. 2011;18:108-28.

38. Wang C, Ding M, Kluger BM. Change in intraindividual variability over time as a key metric for defining performance-based cognitive fatigability. Brain Cogn. 2014;85:251-8.

39. Morrow SA, Rosehart H, Johnson AM. Diagnosis and quantification of cognitive fatigue in multiple sclerosis. Cogn Behav Neurol. 2015;28:27-32.

40. Claros-Salinas D, Dittmer N, Neumann M, et al. Induction of cognitive fatigue in MS patients through cognitive and physical load. Neuropsychol Rehabil. 2013;23:182-201.

41. Neumann M, Sterr A, Claros-Salinas D, Gütler R, Ulrich R, Dettmers C. Modulation of alertness by sustained cognitive demand in MS as surrogate measure of fatigue and fatigability. J Neurol Sci. 2014;340:178-82.
42. Berard JA, Fang Z, Walker LAS, et al. Imaging cognitive fatigue in multiple sclerosis: Objective quantification of cerebral blood flow during a task of sustained attention using ASL perfusion fMRI. Brain Imaging Behav. 2019. doi: https://doi.org/10. 1007/s11682-019-00192-7.

43. Cehelyk EK, Harvey DY, Grubb ML, et al. Uncovering the association between fatigue and fatigability in multiple sclerosis using cognitive control. Mult Scler Relat Disord. 2019;27:269-75.

44. Kluckow SW, Rehbein J, Schwab M, Witte OW, Bublak P. What you get from what you see: parametric assessment of visual processing capacity in multiple sclerosis an its relation to cognitive fatigue. Cortex. 2016;83:167-80.

45. Hoffman S, Tittgemeyer M, von Cramon DY. Cognitive impairment in multiple sclerosis. Curr Opin Neurol. 2007;20:275-80.

46. Chinnadurai SA, Venkatesan SA, Shankar G, Samivel B, Ranganathan LN. A study of cognitive fatigue in multiple sclerosis with novel clinical and electrophysiological parameters utilizing the event related potential P300. Mult Scler Relat Disord. 2016;10:1-6.

47. Hu M, Muhlert N, Robertson N, Winter M. Perceived fatigue and cognitive performance change in multiple sclerosis: uncovering predictors beyond baseline fatigue. Mult Scler Relat Disord. 2019;32:46-53.

48. Lou JS. Physical and mental fatigue in Parkinson's disease: epidemiology, pathophysiology and treatment. Drugs Aging. 2009;26:195-208.

49. Jordan B, Schweden TLK, Mehl T, Menge U, Zierz S. Cognitive fatigue in patients with myasthenia gravis. Muscle Nerve. 2017;56:449-57.

50. Möller MC, de Boussard NC, Oldenburg C, Bartfai A. An investigation of attention, executive, and psychomotor aspects of cognitive fatigability. J Clin Exp Neuropsychol. 2014;36:716-29.

51. Berard JA, Smith AM, Walker LAS. Predictive models of cognitive fatigue in multiple sclerosis. Arch Clin Neuropsychol. 2018;17:17.

52. Aldughmi M, Huisinga J, Lynch SG, Siengsukon CF. The relationship between fatigability and sleep quality in people with multiple sclerosis. Mult Scler J Exp Transl Clin. 2016;2:1-9.

53. Touzet C. Cognitive fatigue: an impaired cortical inhibitory replenishment. Brain Inj. 2017;31:1625-31. 
54. Wylie GR, Flashman LA. Understanding the interplay between mild traumatic brain injury and cognitive fatigue: models and treatments. Concussion. 2017;2:CNC50.

55. Chaudhuri A, Behan PO. Fatigue and basal ganglia. J Neurol Sci. 2000;179:34-42.

56. Chaudhuri A, Behan PO. Fatigue in neurological disorders. Lancet. 2004;363:978-88.

57. Forwell SJ, Brunham S, Tremlett H, Morrison W, Oger J. Primary and nonprimary fatigue in multiple sclerosis. Int J MS Care. 2008;10:14-20.

58. Clarke AL, Delano-Wood L, Sorg SF, Werhane ML, Hanson KL, Schiehser DM. Cognitive fatigue is associated with reduced anterior internal capsule integrity in veterans with history of mild to moderate traumatic brain injury. Brain Imaging Behav. 2017;11:1548-54.

59. Andreasen AK, Iversn P, Marstrand L, Siersma V, Siebner HR, Sellebjerg F. Structural and cognitive correlates of fatigue in progressive multiple sclerosis. Neurol Res. 2019;41:168-76.

60. Möller MC, Nordin LE, Bartfai A, Julin P, Li T-Q. Fatigue and cognitive fatigability in mild traumatic brain injury are correlated with altered neural activity during vigilance test performance. Front Neurol. 2017;8:1-13.

61. Stefancin P, Govindarajan ST, Krupp L, Charvet L, Duong TQ. Resting-state functional connectivity networks associated with fatigue in multiple sclerosis with early age onset. Mult Scler Relat Disord. 2019;31:101-5.

62. Ormstad H, Aass HC, Amthor KF, et al. Serum cytokine and glucose levels as predictors of poststroke fatigue in acute ischemic stroke patients. J Neurol. 2011;258:670-6.

63. Heesen C, Nawrath L, Reich C, Bauer N, Schulz KH, Gold SM. Fatigue in multiple sclerosis: an example of cytokine mediated sickness behaviour? J Neurol Neurosurg Psychiatry. 2006;77:34-9.

64. Lou J. Fatigue in Parkinson's disease and potential interventions. NeuroRehabilitation. 2015;37:25-34.

65. Dobryakova E, Genova HM, DeLuca J, Wylie GR. The dopamine imbalance hypothesis of fatigue in multiple sclerosis and other neurological disorders. Front Neurol. 2015;6:1-8.

66. Wang C, Trongnetrpunya A, Samual IBH, Ding M, Kluger BM. Compensatory neural activity in response to cognitive fatigue. J Neurosci. 2016;36:3919-24.
67. Samuel IBH, Wang C, Burke SE, Kluger BM, Ding M. Compensatory neural responses to cognitive fatigue in young and older adults. Front Neural Circuits. 2019;13:1-12.

68. Johansson B, Wentzel A-P, Andréll P, et al. Evaluation of dosage, safety and effects of methylphenidate on post-traumatic brain injury symptoms with a focuse on mental fatigue and pain. Brain Inj. 2014;28:304-10.

69. Mendonça DA, Menezex K, Jog MS. Methylphenidate improves fatigue scores in Parkinson disease: a randomized controlled trial. Mov Disord. 2007;22:2070-6.

70. Ouellet M-C, Morin CM. Efficacy of cognitive-behavioral therapy for insomnia associated with traumatic brain injury: a single-case experimental design. Arch Phys Med Rehabil. 2007;88:1581-92.

71. Ulrichsen KM, Kaufmann T, Dørum ES, et al. Clinical utility of mindfulness training in the treatment of fatigue after stroke, traumatic brain injury and multiple sclerosis: a systematic literature review and meta-analysis. Front Psychol. 2016;7:1-11.

72. Wendebourg MJ, Heesen C, Finlayson M, Meyer B, Pöttgen J, Köpke S. Patient education for people with multiple sclerosis-associated fatigue: a systematic review. PLoS ONE. 2017;12:e0173025.

73. Ghahari S, Packer T. Effectiveness of online and face-to-face fatigue self-management programmes for adults with neurological conditions. Disabil Rehabil. 2012;34:564-73.

74. Motl RW, Sandroff BM, DeLuca J. Exercise training and cognitive rehabilitation: a symbiotic approach for rehabilitating walking and cognitive functions in multiple sclerosis? Neurorehabilit Neural Repair. 2015;30:499-511.

75. Asano M, Finlayson ML. Meta-analysis of three different types of fatigue management interventions for people with multiple sclerosis: exercise, education and medication. Mult Scler Int. 2014;2014:1-12.

76. Sinclair KL, Ponsford JL, Taffe J, Lockley SW, Rajaratnam SMW. Randomized controlled trial of light therapy for fatigue following traumatic brain injury. Neurorehabil Neural Repair. 2014;28:303-13.

77. Schoenberger NE, Shif SC, Esty ML, Ochs L, Matheis RJ. Flexyx neurotherapy system in the treatment of traumatic brain injury: an initial evaluation. J Head Trauma Rehabil. 2001;16:260-74.

78. Cantor JB, Ashman T, Bushnik T, et al. Systematic review of interventions for fatigue after traumatic 
brain injury: a NIDRR Traumatic Brain Injury Model Systems study. J Head Trauma Rehabil. 2014;29:490-7.

79. Ruiz ML, Sospedra M, Arce SA, Tejeiro-Martinez J, Benito-León J. Current evidence on the potential therapeutic applications of transcranial magnetic stimulation in multiple sclerosis: a systematic review of the literature. Neurologia. 2018. https:// doi.org/10.1016/j.nrl.2018.03.023.

80. León Ruiz M, Rodríguez Sarasa ML, Sanjuá Rodríguez L, Benito-León J, García-Albea Ristol E, Arce Arce S. Current evidence on transcranial magnetic stimulation and its potential usefulness in poststroke neurorehabilitation: Opening new doors to the treatment of cerebrovascular disease. Neurologia. 2018;33:459-72.

81. Sander C, Eling P, Hanken K, Klein J, Kastrup A, Hildebrandt $\mathrm{H}$. The impact of MS-related cognitive fatigue on future brain parenchymal loss and relapse: a 17-month follow-up study. Front Neurol. 2016;7:1-10.

82. Moher D, Liberati A, Tetzlaff J, Altman DG, Group TP. Preferred reporting items for systematic reviews and meta-analyses: the PRISMA Statement. Open Med. 2009;3:123-30.

83. Liberati A, Altman DG, Tetzlaff J, et al. The PRISMA statement for reporting systematic reviews and meta-analyses of studies that evaluate health care interventions: explanation and elaboration. PLOS Med. 2009;6:e1000100.

84. Moher D, Shamseer L, Clarke M, et al. Preferred reporting items for systematic review and metaanalysis protocols (PRISMA-P) 2015 statement. Syst Rev. 2015;4:1-9.

85. Stewart LA, Clarke M, Rovers M, et al. Preferred reporting items for a systematic review and metaanalysis of individual participant data: the PRISMAIPD Statement. J Am Med Assoc. 2015;313:1657-65.

86. Higgins JPT, Altman DG, Gøtzsche PC, et al. The Cochrane Collaboration's tool for assessing risk of bias in randomised trials. BMJ. 2011;343:1-9.

87. Morrow SA, Rosehart H, Johnson AM. The effect of Fampridine-SR on cognitive fatigue in a randomized double-blind crossover trial in patients with MS. Mult Scler Relat Disord. 2017;11:4-9.

88. Fiene M, Rufener KS, Kuehne M, Matzke M, Heinze H-J, Zaehle T. Electrophysiological and behavioral effects of frontal transcranial direct current stimulation on cognitive fatigue in multiple sclerosis. J Neurol. 2018;265:607-17.
89. Kurtzke JF. Rating neurologic impairment in multiple sclerosis: an expanded disability status scale (EDSS). Neurology. 1983;33:1444-52.

90. van Diemen HA, Polman CH, van Dongen TM, et al. The effect of 4-aminopyridine on clinical signs in mutiple sclerosis: a randomized, placebo-controlled, double-blind, cross-over study. Ann Neurol. 1992;32:123-30.

91. Bever CT Jr, Young D, Anderson PA, et al. The effecst of 4-aminopyridine in multiple sclerosis patients: results of a randomized, placebo-controlled, double-blind, concentration-controlled, crossover trial. Neurology. 1994;44:1054-9.

92. Schwid SR, Petrie MD, McDermott MP, Tierney DS, Mason DH, Goodman AD. Quantitative assessment of sustained-release 4-aminopyridine for symptomatic treatment of multiple sclerosis. Neurology. 1997;48:(4):817-21.

93. Stefoski D, Davis FA, Fitzsimmons WE, Luskin SS, Rush J, Parkhurst GW. 4-aminopyridine in multiple sclerosis: prolonged administration. Neurology. 1991;41:1344-8.

94. Blight AR. Effect of 4-aminopyridine on axonal conduction-block in chronic spinal cord injury. Brain Res Bull. 1989;22:47-52.

95. Shi R, Kelly TM, Blight AR. Conduction block in acute and chronic spinal cord injury: different doseresponse characteristics for reversal by 4-aminopyridine. Exp Neurol. 1997;148:495-501.

96. Tecchio F, Cancelli A, Cottone C, et al. Brain plasticity effects of neuromodulation against multiple sclerosis fatigue. Front Neurol. 2015;6:141.

97. Tecchio F, Cancelli A, Cottone C, et al. Multiple sclerosis fatigue relief by bilateral somatosensory cortex neuromodulation. J Neurol. 2014;261:1552-8.

98. Cancelli A, Cottone C, Giordani A, et al. Personalized, bilateral whole-body somatosensory cortex stimulation to relieve fatigue in multiple sclerosis. Mult Scler J. 2018;24:1366-74.

99. Ferrucci R, Vergari M, Cogiamanian F, et al. Transcranial direct current stimulation (tDCS) for fatigue in multiple sclerosis. NeuroRehabilitation. 2014;34:121-7.

100. Schwid SR, Tyler CM, Scheid EA, Weinstein A, Goodman AD, McDermott MP. Cognitive fatigue during a test requiring sustained attention: a pilot study. Mult Scler. 2003;9:503-8.

101. Goodman AD, Brown TR, Krupp LB, et al. Sustainedrelease oral fampridine in multiple sclerosis: a 
raondomized, double-blind, controlled trial. Lancet Neurol. 2009;373:732-8.

102. Parmenter BA, Denney DR, Lynch SG. The cognitive performance of patients with multiple sclerosis during periods of high and low fatigue. Mult Scler. 2003;9:111-8.

103. Paul RH, Beatty WW, Schneider R, Blanco CR, Hames KA. Cognitive and physical fatigue in multiple sclerosis: relations between self-report and objective performance. Appl Neuropsychol. 1998;5:143-8.

104. Ayache SS, Chalah MA. Transcranial direct current stimulation: a glimmer of hope for multiple sclerosis fatigue? J Clin Neurosci. 2018;55:10-2.

105. Berard JA, Smith AM, Walker LAS. Predictive models of cognitive fatigue in multiple sclerosis. Arch Clin Neuropsychol. 2019;34(1):31-8.

106. Clancy M, Drerup M, Sullivan AB. Outcomes of cognitive-behavioural treatment for insomnia on insomnia, depression, and fatigue for individuals with multiple sclerosis. Int $J$ MS Care. 2015;17:261-7.

107. Berard JA, Smith AM, Walker LAS. A longitudinal evaluation of cognitive fatigue on a task of sustained attention in early relapsing-remitting multiple sclerosis. Int J MS Care. 2018;20:55-61.

108. Bruce JM, Bruce AS, Arnett PA. Response variability is associated with self-reported cognitive fatigue in multiple sclerosis. Neuropsychology. 2010;24:77-83.

109. Harrison AM, das Nair R, Moss-Morris R. Operationalising cognitive fatigability in multiple sclerosis: a Gordian knot that can be cut? Mult Scler J. 2017;23:1682-96.

110. Bartolucci AA, Hillegass WB. Overview, strengths, and limitations of systematic reviews and metaanalyses. In: Chiappelli F, et al., editors. Evidencebased practice: toward optimizing clinical outcomes. Berlin: Springer; 2010. p. 17-33. 